\title{
MATEMÁTICA: UM DESAFIO INTERNACIONAL PARA ESTUDANTES SURDOS*
}

\author{
Karen L. Kritzer** \\ Claudia M. Pagliaro ${ }^{* * *}$
}

$\mathrm{H}$ á estudos que indicam que a vasta maioria de estudantes surdos tem desempenho significativamente abaixo da media de sua série e de seus pares ouvintes em matemática (MITCHELL, 2008; TRAXLER, 2000). Essa defasagem tem sido encontrada em diferentes países (LEYBAERT; VAN CUTSEM, 2002; TRAXLER, 2000) e em diferentes conceitos e áreas matemáticas, incluindo o conceito de número, cálculo (TRAXLER, 2000), resolução de problemas e raciocínio (ANSELL; PAGLIARO, 2006; KRITZER, 2009b; NUNES; MORENO, 2002). Além disso, há pesquisas que indicam que esta defasagem começa cedo, antes mesmo do início da escolaridade formal (KRITZER, 2009a; KRITZER; PAGLIARO, 2013) e amplia-se com o passar do tempo (TRAXLER, 2000).

Estas evidências convincentes têm levado os pesquisadores a questionarem as bases do desempenho de tais crianças para definir o detalhamento de tais diferenças e desenvolver intervençôes que atinjam e diminuam a

* Traduzido por Beatriz Vargas Dorneles.

N. do T.: Optou-se por traduzir "deaf and hard-of-hearing students", do original, como "estudantes surdos”, para manter a nomenclatura utilizada no Brasil.

** School of Lifespan Development and Educational Sciences, Kent State University. Kent (Ohio) - Estados Unidos.

*** Dept. of Counseling, Educational Psychology \& Special Education, Michigan State University. East Lansing (Michigan) - Estados Unidos.

Contato com as autoras:<kkritzer@kent.edu>

Cad. Cedes, Campinas, v. 33, n. 91, p. 431-439, set.-dez. 2013

Disponível em <http://www.cedes.unicamp.br> 
defasagem. Neste breve panorama nós descrevemos as pesquisas relacionadas a áreas específicas favoráveis e os desafios que as crianças surdas parecem enfrentar, para então discutirmos os fatores potenciais que têm impacto sobre tal desempenho e, finalmente, descrevemos programas de intervenção que começam a aparecer nesta área de grande carência.

\section{Áreas específicas favoráveis e desafios}

Frente às evidências de que crianças surdas começam o ensino formal já atrás de crianças ouvintes em conceitos matemáticos fundamentais (KRITZER, 2009a; NUNES; MORENO, 1998), Pagliaro e Kritzer (2013) tentaram descrever especificamente como a compreensão matemática se apresenta para pequenas crianças surdas. Vinte crianças com idades entre 3 e 5 anos foram solicitadas a completar um teste padronizado, bem como uma série de tarefas não padronizadas que refletiam o desenvolvimento típico nas áreas de número, geometria, medidas, resolução de problemas, padrões, raciocínio e álgebra, definidos para crianças ouvintes (DEVELOPMENTAL TRACKER, 2001; SARAMA; CLEMENTS, 2009). Os resultados mostraram subconceitos dentro de cada área, com diferentes níveis de desempenho das crianças (médio, acima ou abaixo do esperado para sua faixa etária), dado certo nível de ajuda. Os resultados apresentaram áreas de desempenho relativamente superior (geometria) e de desempenho inferior (medidas e resolução de problemas) ao de crianças ouvintes. Vários conceitos mostraram-se particularmente desafiadores para crianças surdas de diferentes idades, tais como estimativa, tempo e sequenciamento, áreas nas quais já haviam sido encontradas dificuldades em estudos com adultos e crianças mais velhas (BULL et al., 2011; NUNES; MORENO, 2002). Então, é possível dizer que as fragilidades apresentadas pelas crianças surdas em conhecimentos e habilidades matemáticas básicas formam a base para as dificuldades encontradas entre estudantes mais velhos e adultos. Entretanto, pode ser, também, que crianças surdas não desenvolvam conceitos matemáticos da mesma forma que as crianças ouvintes e, dessa forma, não deveriam ser medidas com o mesmo padrão usado para crianças ouvintes (ANSELL; PAGLIARO, 2006).

Uma considerável parcela de pesquisa tem sido desenvolvida voltada para as estratégias e soluções utilizadas por crianças surdas na resolução de problemas. Tais estudos têm variado quanto à forma de apresentação 
dos problemas, ou seja, pela modalidade de linguagem: escrita (HYDE; ZEVENBERGEN; POWER, 2003; KELLY; MOUSLEY, 2001) ou de sinais (ANSELL; PAGLIARO, 2006; PAGLIARO; ANSELL, 2012), e pela forma e série/idade: equações numéricas (FROSTAD, 1999) ou resolução de problemas com histórias (ANSELL; PAGLIARO, 2006; KELLY; MOUSLEY, 2001). Independentemente da apresentação, os achados têm sido semelhantes e indicam que: a) estudantes surdos têm desempenho inferior a crianças ouvintes na resolução de problemas; b) tanto o nível de leitura como o nível de "adivinhação" no problema afetam o sucesso (isto é, quanto melhor o nível de leitura, maior o sucesso; quanto maior a "adivinhação", menor o sucesso); c) existem semelhanças com as crianças ouvintes quanto às estratégias de solução, apesar de existirem diferenças no desenvolvimento das estratégias.

Fatores que contribuem para o desempenho

Estudos mostram que o desempenho na matemática não é inerente à surdez ou a sua capacidade de entender conceitos matemáticos, mas parece estar mais ligado a fatores externos tais como a formação de professores, bem como a oportunidades educacionais e a fatores externos à sala de aula (PAGLIARO; ANSELL, 2002; KRITZER, 2008; PAGLIARO; KRITZER, 2010; PAGLIARO, 2010).

Já está bem documentado que o conhecimento do conteúdo da área entre os professores influencia o ensino e que professores de estudantes surdos têm uma formação frágil em matemática e educação matemática (KELLY, LANG; PAGLIARO, 2003; PAGLIARO, 1998). Pesquisas têm mostrado que o conteúdo insuficiente, bem como uma frágil formação pedagógica podem influenciar o ensino e têm impacto sobre a aprendizagem dos estudantes. Por exemplo, Pagliaro e Ansell (2002) mostraram que professores do ensino básico que tiveram um ou mais cursos em métodos de ensino de matemática eram mais propensos a incluir problemas com histórias no seu ensino. Kelly, Lang e Pagliaro (2003) também mostraram uma diferença significativa no comportamento de ensino de professores de surdos que tiveram cursos de capacitação em áreas relacionadas à matemática, em comparação com aqueles que não tiveram, com os primeiros apresentando uma qualificação melhor e um ensino mais baseado na cognição. Lang e Pagliaro (2007) confirmaram estes resultados, mostrando os benefícios da 
formação em matemática, na memorização de conceitos geométricos e variáveis preditoras. Um estudo, entretanto, apresentou resultados contrários. No trabalho de Pagliaro e Kritzer (2005), nenhuma diferença significativa foi encontrada na prática instrucional entre aqueles professores com e sem formação formal em matemática/educação matemática. Entretanto, os autores do estudo ressaltam que estes resultados exigem mais investigação, já que poucos professores na amostra tinham formação anterior em ensino matemático.

Professores mal preparados em matemática podem conduzir ao desenvolvimento de poucas oportunidades para o ensino de matemática de qualidade na sala de aula. Tanto Kelly, Lang e Pagliaro (2003) como Pagliaro e Ansell (2002) encontraram evidências de que os professores de surdos proveem relativamente poucas oportunidades para a solução de problemas durante o ensino de matemática. Mais ainda, Pagliaro e Kritzer (2005) mostraram que professores de crianças surdas falham não só em prover ensino matemático significativo e centrado nos estudantes como recomendado na área, mas também não o fazem devido à baixa expectativa que têm quanto aos seus alunos. Não são somente as oportunidades de aprendizagem (presentes ou ausentes) dentro da sala de aula que afetam a compreensão dos estudantes, mas também as oportunidades fora da sala de aula, incluindo o meio familiar, que podem contribuir para o conhecimento de conceitos fundamentais.

$\mathrm{Na}$ análise das interações de pais com crianças pequenas surdas, de 3 a 5 anos, Kritzer $(2008 ; 2012)$ encontrou evidências de que as crianças que tiveram escores relativamente altos no desempenho matemático tendiam a passar mais tempo envolvidas em interaçôes significativas com familiares adultos. Elas também estavam mais expostas e com maior frequência a conceitos matemáticos básicos, tais como número, quantidade, tempo/ sequência e classificação, do que aquelas crianças com desempenho relativamente inferior em matemática. As diferenças na mediação parental de crianças com "alto" e "baixo" desempenhos existiam mesmo quando era dada, aos pais, uma tarefa a realizar que era de natureza matemática. Em uma análise complementar dos dados, Pagliaro e Kritzer (2010) também concluíram que o comportamento de mediação entre os pais e seus filhos surdos poderia ter contribuído para o desempenho das crianças na matemática. Estes autores analisaram a interação de seis pais com suas crianças pequenas (três com habilidades numéricas relativamente altas e três com habilidades numéricas relativamente baixas) em vários comportamentos 
apresentados para facilitar a aprendizagem (FEUERSTEIN; RAND, 1997). Os resultados não só mostraram uma diferença entre os dois grupos (com altas e baixas habilidades numéricas) quanto à frequência na qual os pais e a criança exibiam comportamentos de aprendizagem, mas também na qualidade de tais comportamentos. Os pais e seus filhos com relativamente altas habilidades em matemática tendiam a estender o discurso através de explicações e questionamentos adicionais. Estas crianças eram também mais aptas a coordenar dois ou mais comportamentos de aprendizagem, tais como iniciar a comunicação, comparar e referenciar o tempo. Então, os resultados sugerem uma possível relação entre comportamentos de aprendizagem precoces, mediação no lar e habilidades matemáticas precoces.

\section{Programas de intervenção}

Uma formação melhor para professores que ensinarão crianças surdas inclui conhecimento de como apresentar matemática conceitualmente embasada em resolução de problemas, fundamentais em salas de aula com estudantes surdos. Estas salas de aula deveriam priorizar a integração de oportunidades significativas do mundo real mais do que aprendizagem procedural que não beneficia estudantes a longo prazo. Também é importante que intervenções comecem muito cedo na família para ajudar as crianças a desenvolverem os conceitos iniciais antes da escola. Um programa de intervenção (KRITZER; PAGLIARO, 2012) está fazendo justamente isto: trabalhando com famílias para tirar as crianças de um início difícil na compreensão matemática, mostrando aos pais como interagir com suas crianças surdas, de forma que estimulem o desenvolvimento cognitivo e promovam o uso natural e significativo da matemática em casa. Resultados deste estudo indicam mudanças produtivas nos comportamentos paternos que apontam um efeito positivo no conhecimento, no reconhecimento e na mediação dos pais em conceitos matemáticos iniciais com seus filhos surdos (idem, ibid.).

Outras intervenções que incluíram crianças surdas em idade escolar apresentaram resultados encorajadores. Nunes e Moreno (2002) organizaram um programa de intervenção destinado a desenvolver a compreensão da numeralização em crianças surdas. Os resultados positivos atingidos foram atribuídos, em parte, à organização do programa que estimulou o acesso de estudantes surdos ao currículo matemático. Tais estudos, incluindo alguns 
endereçados às necessidades de estudantes mais velhos (MOUSLEY; KELLY, 1998), mostram que a intervenção é necessária e é um elemento que potencialmente contribui para o sucesso dos estudantes.

\section{Olhando para o futuro}

Embora os itens anteriores provenham informação valiosa quanto à compreensão matemática em pessoas surdas, existe muito ainda que é desconhecido. Entre os tópicos que necessitam de mais investigação estão os seguintes:

- Necessidade de determinar se as crianças surdas seguem a mesma trajetória de desenvolvimento dos conceitos matemáticos e habilidades já estabelecidas para crianças ouvintes e, se não, qual é o padrão normal de desenvolvimento para esta população;

- Avaliação contínua de crianças surdas, jovens e adultos que tiveram sucesso na matemática e na resolução de problemas para determinar os fatores que contribuíram para tal;

- Desenvolvimento e testagem de intervenções para determinar as melhores práticas para a educação matemática de crianças surdas;

- Avaliação de possível impacto do desempenho e compreensão matemática em outras disciplinas, incluindo Leitura e Ciências.

Nossa intenção aqui não foi prover uma visão abrangente de toda a pesquisa que está sendo desenvolvida em educação matemática e surdez outros estudiosos têm feito isso e remetemos os leitores a eles (GOTTARDIS; NUNES; LUNT, 2011; PAGLIARO, 2010) -, mas sim iluminar algumas áreas e prover algum contexto para pesquisas futuras. Nós esperamos que esse número temático possa desencadear um interesse mais amplo pela área.

\section{Referências}

ANSELL, E.; PAGLIARO, C. The relative difficulty of signed arithmetic story problems for primary level deaf and hard-of-hearing students. Journal of Deaf Studies and Deaf Education, v. 11, n. 2, p. 153-170, 2006.

BULL, R. et al. Numerical estimation in deaf and hearing adults. Learning and Individual Differences, n. 21, p. 453-457, 2011. 
DEVELOPMENT TRACKER. Retrieved Oct. 29, 2011. From: <http:// www.pbs.org/parents/childdevelopmenttracker/index.html>

FEUERSTEIN, R.; RAND, Y. Don't accept me as I am: helping retarded performers excel. Arlington Heights, IL: Skylight, 1997.

FROSTAD, P. Deaf children's use of cognitive strategies in simple arithmetic problems. Educational Studies in Mathematics, n. 40, p. 129-153, 1999.

GERSTEN, R. et al. Universal screening in mathematics for the primary grades: Beginnings of a research base. Exceptional Children, v. 78, n. 4, p. 423-445, 2012.

GOTTARDIS, L.; NUNES, T.; LUNT, I. A synthesis of research on deaf and hearing children's mathematical achievement.Deafness \& Education International, v. 13, n. 3, p. 131-150, 2011.

HYDE, M.; ZEVENBERGEN, R.; POWER, D. Deaf and hard of hearing students' performance on arithmetic word problems. American Annals of the Deaf, v. 148, n. 1, p. 56-64, 2003.

KELLY, R.; LANG, H.; PAGLIARO, C. Mathematics word problem solving for deaf students: a survey of perceptions and practices in grades 6-12. Journal of Deaf Studies and Deaf Education, v. 8, n. 2, p. 104-119, 2003.

KELLY, R.; MOUSLEY, K. Solving word problems: more than reading issues for deaf students.American Annals of the Deaf, v. 146, n. 3, p. 251262, 2001.

KRITZER, K. Family mediation of mathematically based concepts while engaged in a problem-solving activity with their young deaf children. The Journal of Deaf Studies and Deaf Education, v. 13, n. 4, p. 503-517, 2008.

KRITZER, K. Barely started and already left behind: a descriptive analysis of the mathematics ability demonstrated by young deaf children. Journal of Deaf Studies and Deaf Education, v. 14, n. 4, p. 409-421, 2009a.

KRITZER, K. Families with young deaf children and the mediation of mathematically-based concepts within a naturalistic environment. American Annals of the Deaf, v. 153, n. 5, p. 474-483, 2009 b.

KRITZER, K. The story of an outlier: a case study of one young deaf 
Matemática: um desafio internacional para estudantes surdos

child and is journey towards early mathematical competence. Deafness and Education International, v. 14, n. 2, p. 1-9, 2012.

KRITZER, K.L.; PAGLIARO, C.M. An intervention for early mathematics success: Building Math Readiness Parents as Partners Project, Phase 1 - Outcomes. Journal of DeafStudies and Deaf Education, v. 17, n. 4, 2012.

LEYBAERT, J.; VAN CUTSEM, M.-N. Counting in sign language. Journal of Experimental Child Psychology, n. 81, p. 482-501, 2002.

LANG, H.; PAGLIARO, C. Factors predicting recall or mathematics terms by deaf students: Implications for teaching. Journal of Deaf Studies and Deaf Education, v. 12, n. 4, p. 449-460, 2007.

MITCHELL, R. Academic achievement of deaf students. In: JOHNSON, R.; MITCHELL, R. (Ed.). Testing deaf students in an age of accountability. Washington, DC: Gallaudet University Press, 2008. p. 38-50.

MOUSLEY, K.; KELLY, R. Problem solving strategies for teaching mathematics to deaf students. American Annals of the Deaf, v. 143, n. 4, p. 325-336, 1998.

NUNES T.; MORENO, C. An intervention program for promoting deaf pupils' achievement in mathematics. Journal of Deaf Studies and Deaf Education, v. 7, n. 2, p. 120-133, 2002.

NUNES, T.; MORENO, C. Is hearing impairment a cause of difficulties in learning mathematics? In: DONLAN, C. (Ed.). The development of mathematical skills: studies in developmental psychology. Hove, UK: Psychology Press, 1998. p. 227-254.

PAGLIARO, C. Mathematics preparation and professional development of deaf education teachers. American Annals of the Deaf, v. 143, n. 5, p. 373379, 1998.

PAGLIARO, C. Mathematics instruction and learning of deaf/hard-ofhearing students: What do we know? Where do we go? In: MARSCHARK, M.; SPENCER, P. (Ed.). Oxford handbook of deaf studies, language and education. v. 2. New York: Oxford University Press, 2010. p. 156-171.

PAGLIARO, C.; ANSELL, E. Story problems in the deaf education classroom: frequency and mode of presentation. Journal of DeafStudies and Deaf Education, n. 7, p. 107-119, 2002. 
PAGLIARO, C.; ANSELL, E. Deaf and hard-of-hearing students' problem-solving strategies with signed arithmetic story problems. American Annals of the Deaf, v. 156, n. 5, p. 438-458, 2012.

PAGLIARO, C.; KRITZER, K. Discrete mathematics in deaf education: a survey to determine knowledge and use. American Annals of the Deaf, v. 150, n. 3, p. 251-259, 2005.

PAGLIARO, C.; KRITZER, K. Learning to learn: an analysis of early learning behaviors demonstrated by young deaf/hard-of-hearing children with high/low mathematics ability. Deafness and Education International, v. 12, n. 10, p. 2-25, 2010.

PAGLIARO, C.; KRITZER, K. The math gap: an analysis of the mathematics performance of deaf/hard-of-hearing preschool children. Journal of Deaf Studies and Deaf Education, v. 18, n. 2, p. 139-160, 2013.

SARAMA, J.; CLEMENTS, D. Early childhood mathematics education research: learning trajectories for young children. New York: Routledge, 2009.

TRAXLER, C.B. The Stanford Achievement Test, 9th Edition: national norming and performance standards for 13 deaf and hard-of-hearing students. Journal of Deaf Studies and Deaf Education, v. 5, n. 4, p. 337-348, 2000 . 